\title{
Emotions Are the Rudder of Our lives Intensity and Duration in Young Adults ${ }^{\dagger}$
}

\author{
Marinella Coco ${ }^{1, *}$, Maria Cristina Petralia ${ }^{1,2}$, Giulia Di Gregorio ${ }^{2}$, Andrea Buscemi ${ }^{3}$ and \\ Valentina Perciavalle ${ }^{2}$ \\ 1 Department of Biomedical and Biotechnological Sciences, University of Catania, 95123 Catania, Italy; \\ marinella.coco@gmail.com \\ 2 Department of Educational Sciences, University of Catania, 95124 Catania, Italy; \\ m.cristinapetralia@gmail.com (M.C.P.); giuliadigregor@gmail.com (G.D.G.); valentinaperciavalle@unict.it (V.P.) \\ 3 Horus Cooperative Social, 97100 Ragusa, Italy; andreabuscemi@virgilio.it \\ * Correspondence: marinella.coco@gmail.com or marinella.coco@unict.it; Tel.: +39-338-153-6579 \\ + Presented at the International and Interdisciplinary Conference IMMAGINI? Image and Imagination \\ between Representation, Communication, Education and Psychology, Brixen, Italy, 27-28 November 2017. \\ Published: 22 November 2017
}

\begin{abstract}
The present study aims to expand knowledge about emotions by studying their intensity and duration in young adults. We showed two different videos in two different days to a total of 103 first-year students from university of Catania. Through these videos, we tried to elicit medium intensity emotion to emulate everyday situations: watching television news or short internet video, seeing advertisement of humanitarian associations to sensitise people. The aim was to see if the videos could influence the emotional state of the students for two hours.
\end{abstract}

Keywords: emotions, Profile of Mood States, personality, education

\section{Introduction}

Today more than ever, we are constantly surrounded by many stimuli that attracts our attention and changes our emotional state: from a song that makes us happy to listen to it, to a beggar in the streets, that can make us feel uncomfortable and raise negative emotion such as anxiety or sadness. Our emotions swing naturally and constantly, and are part of our daily life.

But what is exactly an "emotion"? There have been many definition thought history, starting from the ancient Greeks philosopher Plato, that thought of emotions as "uncontrollable forces in contrast with reason", and Aristotle, which affirmed that "emotions are reaction to our way of interpreting the world" [1]. After them, many other philosophers speculated over the nature of emotions, such as René Descartes or John Lock, but only on a theoretical level; emotions will start to be studied through biological perspective in XIXth century with Charles Darwin and in the second half of the century with William James and his essay "What is an emotion" published in 1884 on the periodical "Mind". The idea of what is an emotion developed and modified in time bringing us to the present conception, that thinks emotions are made up of multiple factors, such as physiological changes, action readiness, current concern and conscious experience [2].

Many scientists, doctors, psychologist studied the nature of emotion and the physiological and cognitive patterns associated with every one of them; thanks to those researches, our knowledge on emotion has significantly increased. Different studies underlined we should distinguish between different emotional phenomena: sensation, feelings, emotions, and mood. Although there is quite a confusion between these terms in scientific literature, many believes they should be distinguished. APA (American Psychology Association) defines sensation as "The process by which stimulation of a sensory receptor gives rise to neural impulses that result in an experience, or awareness of, 
conditions inside or outside the body." Feelings are often confused with sensation, as they both are experienced through outside stimuli reacting with our senses, but the fundamental difference between them is that feelings originates from the inside while sensations derive from outside. As A. Damasio, professor of neuroscience at The University of California says, "Feelings are mental experiences of body states, which arise as the brain interprets emotions, themselves physical states arising from the body's responses to external stimuli. (The order of such events is: I am threatened, experience fear, and feel horror.)" Between sensation and feeling there is also a distinction of time; before the feeling can arise, the electric impulse from the peripheric sensors must arrive to the brain, that elaborates it and gives origin to the feeling of something. For example, "First is the sensation of touch, sensation then recedes, and the feeling of heat or cold arises. The element of time must separate sensation and feeling, or else what we call feeling would probably not exist" [3-6].

As far as emotion and mood are concerned, "a broad range of distinctions is proposed, ranging from physiological and neurological through to behavioural and social criteria" [7].

Panksepp [8] distinguish them from a neurological point of view, they have, if fact, different neural patterns. Wierzbicka [9] talks about semantic distinction between the words. Beddie et al. [7] asked non-academic population if and how would they make a distinction between them. Results showed that "emotion is less controllable than mood, emotions are more intense, brief, volatile, etc. than moods".

The definition of emotion for APA is "A complex pattern of changes, including physiological arousal, feelings, cognitive processes, and behavioral reactions, made in response to a situation perceived to be personally significant".

Many studies questioned already emotion duration and intensity under different circumstances like [10]. What they found out is that "the higher the importance of the emotion-eliciting situation and the higher the intensity of the emotion at onset, the longer the emotional experience lasts" [11].

The present study aims to expand knowledge about emotions by studying their intensity and duration in young adults. We showed to two groups of first-year students from university of Catania two different video: a negative one that showed sad and uncomfortable scenes and a positive one that showed a happy scenario. Through these videos, we tried to elicit medium intensity emotion, like the ones we can experience watching news in television, or a video on the internet. To emulate these kind of everyday situations, we also choose short length video (about $5 \mathrm{~min}$ each).

We studied how the videos affected emotions just after and to two hours later the vision, and how specific personality factors can affect this change. The hypothesis of research is that participants will experience a change in their emotional state after the vision of the videos and that this change will last after two hours, even if with lower intensity. Is there a difference between negative and positive emotion retaining? We also took digit radio of participants (hand photocopy) to see in there is a relation between prenatal androgen, that increase risk attitude, promoting faster visual-motor reactions [12] and emotion retaining.

\section{Matherial and Method}

\subsection{Participants}

200 students enrolled in the first year of psychology's faculty at the university of Catania were informed of the research and its modalities. They were asked to come to come to university in two different days to administrate two different video: a sad one on the first day and a happy one on the second day. 38 students came to the first encounter and 65 to the second, for a total of 103 participants, of which 12 males and 91 females. We also asked them photocopy of both their hands in order to calculate ratio between second and fourth finger (digit radio).

The subjects were fully informed of the purpose of this study and possible risks before signing the informed consent from in accordance with the ethical standards laid down in the 1964 Declaration of Helsinki and the procedures have complied with the ethical guidelines and code of conduct of the APA and AIP (Italian Psychological Association). No approval from Ethic Committee was necessary for the following study. 


\subsection{Protocol}

The experiment was conducted in a university classroom in two different days with 1 week gap. Participants were asked to fill in different tests and to watch a video, after which they filled again two test. The test administration during the two different days was the same, only the content of the video changed. All tests were self-administered.

First thing (Figure A1) participants were asked to fill in a Big Five Questionnaire-2 and a copy of POMS (Profile of Mood States). This first step was in order to evaluate the personality and the actual mood state of each person. After this, using the room projector, a short video was played. For the first encounter a "sad video" was played, that showed images of war, violence and injustice in combination with a slow piano music (the video cans be seen at https://www.youtube.com/watch?v=QC6Zw_w_1TE, the accompaniment was "Cold" by J. Méndez); for the second encounter, we projected a "happy video" that consisted of a man dancing and signing of joy (I'm signing in the rain-Gene Kelly). The two videos had similar duration: 5 min the first, 4:40 the second. Immediately after the vision, participants were asked to fill in a State-Trait Anxiety Inventory Y (STAI-Y) and to re-fill a POMS. After this, participants were dismissed asking them to return after two hours to fill in another copy of POMS and to consign the photocopy of their hands.

\subsection{Limits}

The results of this study came from a sample made principally of female: this means it's not possible to estimate if there are statistically significant differences between male and female in terms emotional response to the videos. Another limit is that all the sample was made of students enrolled in the faculty of psychology. To obtain more accurate results it would be beneficial to repeat the study on different faculty's students, as we can suppose that, students enrolled in psychology, have a natural inclination to social relation, which could influence their personality to be more emphatic and emotional.

\subsection{Instruments}

\subsubsection{Big Five Questionnaire-2}

The Big Five Questionnaire-2 [13] is a test based on the theory of a 5 factors personality: extraversion, agreeableness, conscientiousness, emotional stability and mental openness. These different factors do not represent a particular theoretical point of view. They derive from the empirical, factorial analysis of the terms of the common language used to describe ourselves or others. These 5 large dimensions are composed of a set of complementary sub-dimensions: dynamism and dominance for extraversion, cooperativeness/empathy and cordiality/friendly attitude for agreeableness, diligence and perseverance for conscientiousness, emotion control and impulses control for emotional stability, openness to culture and openness to experience for mental openness. The BFQ-2 also has a lie scale (divided in lie egoistic and lie moralistic) to identify overly faux profile that cannot be reliable. The test is composed of 134 items with a Likert scale that goes from 1 (minimum) to 5 (maximum). The Big Five includes excellent internal stability and stability over time. The scales also have good discriminative validity and very consistent results when self-administered.

\subsubsection{POMS-Profile of Mood States}

The Profile of Mood States (POMS) [14] is a tool for the global measurement of moods and their fluctuations (anxiety, depression, confusion, anger, fatigue, vigour and interpersonal relationships).

This is a self-administered pencil-type questionnaire. It takes about 5-10 $\mathrm{min}$ to be filled in and includes 58 items. A scale of Likert of 5 entries allows to collect the answers (of 0 to $5 /$ from "Not at All", "A Little", "Moderately", "Quite a Lot" or "Extremely" to indicate your feeling.). The scale provides 6 sub-scores (stress-anxiety, depression-rejection, anger-hostility, fatigue-inertia, vigour-activity and confusion) and an overall score witch we used in this research (total mood disorder). 


\subsubsection{STAI-Y - State-Trait Anxiety Inventory}

STAI-Y [15] is a commonly used measure of trait and state anxiety and is useful to have a general measure of stress. It has 40 Items, 20 for state anxiety, that refers to the uneasiness felt in specific situation, and 20 for trait anxiety, that refers to a more generic feeling of anxiety and insecurity as a more stable element of personality. Rated on a 4-point scale that goes from 1 (almost never) to 4 (almost always). Subjects answer in terms of intensity of feeling on a 4-point scale that goes from 1 (almost never) to 4 (almost always).

It is self-administered and takes about 15 to $20 \mathrm{~min}$ to be filled in. Considerable evidence attests to the construct and concurrent validity of the scale.

\subsection{Digit Radio}

To detect prenatal androgens, that is the amount of testosterone to which the subjects of the sample were exposed in the womb, the hand test was used. Photocopied hands of the participants were measured in millimetres from meta-carpal-phalangeal crease (PC) to the fingertips (FT); both the index finger (2D) that the ring finger (4D). A ratio greater than 1 indicates that the index is longer ring finger and vice versa [16,17].

It was found that finger length is directly related to the foetus's exposure to testosterone: the greater length of the ring finger compared to the index finger (digit ratio $<1$ ) would indicate elevated level of testosterone to which the child has been exposed during the pregnancy. The index-ring finger length ratio was determined by measuring the right hand only, since it has been shown that this length in the right-hand report would demonstrate greater sexual differences between men and women, and are therefore considered more sensitive to prenatal androgens.

A research carried out by Coates et al. [12] conducted in London, found that the index-ring finger length ratio predicted the long-term revenue-generating of a sample of traders 1 and the number of years that they would remain in the business. The results of this research point out that prenatal androgens increase risk attitude, promoting faster visual-motor reactions resulting in an improved performance of the traders. In fact, research shows that those who had the longest index ring obtained more profit.

\section{Results}

Anxiety evaluation thought STAY-Y (Figure A2), revealed state anxiety values were slightly smaller in confront of trait anxiety values; this means subjects of the sample were in relatively good conditions at the moment of test administration.

Analysis of variance (ANOVA) showed mean value of the sample's anxiety during the negative video administration are 61.17 (S.D. \pm 7.378 ), whereas mean value of the sample's anxiety during the positive video administration are 43.3 (S.D. \pm 11.98 ). Normative scale, included between 20 and 80, shows clearly that the vision of the negative video affects negatively anxiety levels.

Emotion's evaluation through POMS (Figure A3) point out different results for the two videos. For the negative video, right after the vision, mean values strongly increase with $p>0.0001$; after two hours (after) these values decrease again returning to pre-video levels. Statistic correlation confirm that between pre and after video $p>0.9999$.

Emotion's evaluation of the positive video point out a decrease in the sample's mean value. Between pre and post video there's a decrease with $p<0.0001$; between pre and after video there still is a signifi-cant difference as mean values are lower in the after video with $p=0.0004$.

Results moreover, show that subjects most exposed to testosterone during intrauterine life respond bet-ter that the others, this means their emotional change just after the video is more accentuate that the rest of the sample.

Results of BIG 5 and possible correlation between personality factors and changes in emotional state are still under analysis. 


\section{Conclusions}

The initial hypothesis of this study were two: emotions in young adults can be changed and stimulated by the vision of a short video; this change can last up to two hours, even if it will be less noticeable.

We asked participants to come to university in two different days, during which we made them see two different shorts videos (about $5 \mathrm{~min}$ each): a negative one on the first day, a positive on the second. The aim was to stimulate changes in their emotions and to see if they could last up to two hours after the vision of the video. The videos showed respectively images of war and killing on the first day, and a happy man singing and dancing on the second.

Anxiety evaluation thought STAY-Y test confirmed subjects were in relatively good conditions at the moment of test administration.

The comparison between POMS pre-video and POMS post-video confirmed our first hypothesis. Both video influenced anxiety levels and emotions in our sample (Figure A3). The vision of the negative video raised anxiety levels, whereas the vision of the positive one lowered them.

Thanks to the analysis of digit radio, we found out subjects most exposed to testosterone during intrau-terine life respond better that the others $[18,19]$. This means their emotional change just after the video is more accentuate that the rest of the sample. This result suggests further investigations in the future.

The analysis of POMS administered $2 \mathrm{~h}$ after the vision of the videos (we will refer to them as POMS after-video) confirmed our second hypothesis only in part. No statistically relevant difference was found between the POMS pre-video and the POMS after-video concerning the negative video; this means the change in anxiety levels after the vision of the videos went back to initial levels within two hours.

Results of POMS administered with the positive video showed a statistically relevant difference be-tween pre video levels and after video levels. Two hours after the vision of the positive video, anxiety levels were still statistically lower that the pre-video levels. This means the positive video may have ac-tually affected the samples emotions and anxiety levels.

Results show a difference in positive and negative emotion retaining. Negative emotions are less re-tained than positive emotions.

This difference may be linked to defence mechanism (denial, repression, etc.), as we could naturally suppress negative emotions to defend ourselves and foster positive emotions to feel better. As said in the introduction, the videos were chosen to emulate television news, short internet video, long adver-tisement of humanitarian associations that tries to sensitise people and other sorts of audio-visual stimuli that tries to attract interest by stimulating emotions. This difference in emotion retaining may also be accentuate by the strong media exposure people are in contact with. Continuous exposure to new in-formation every day, several times per day, may have lead us to get used to continuously "overwrite" our emotions. This means emotions changes are quick and negative emotion retaining might be influ-enced by this.

Further investigations between continuous media exposure and emotion retaining would be beneficial to deepen this relationship.

Acknowledgments: We want to thank all students who participated in search

Author Contributions: M.C., G.D.G., M.C.P., A.B. and V.P. conceived and designed the experiments; M.C. and G.D.G. performed the experiments; M.C. and A.B. analyzed the data; M.C., G.D.G., A.B. and V.P. contributed analysis tools; M.C., M.C.P., G.D.G., A.B. and V.P. wrote the paper. Authorship must be limited to those who have contributed substantially to the work reported.

Conflicts of Interest: The authors declare no conflict of interest. 


\section{Appendix A}

The sample of psychology students was chosen to evaluate in this particular sample the emotional-emotional response indispensable in the profession. In this regard, attention has been paid to personality traits.

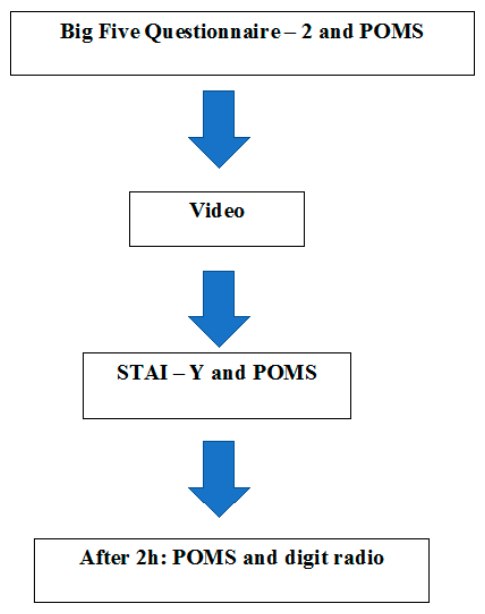

Figure A1. Protocol of research.

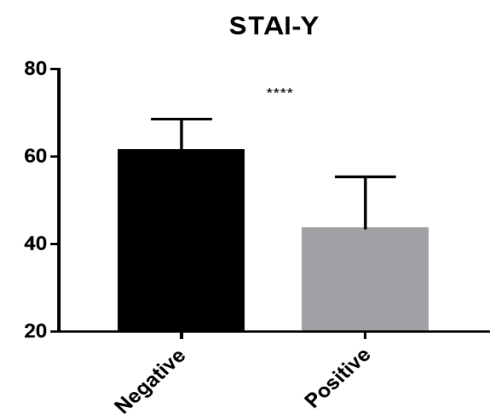

Figure A2. Results at the STAY-Y test.
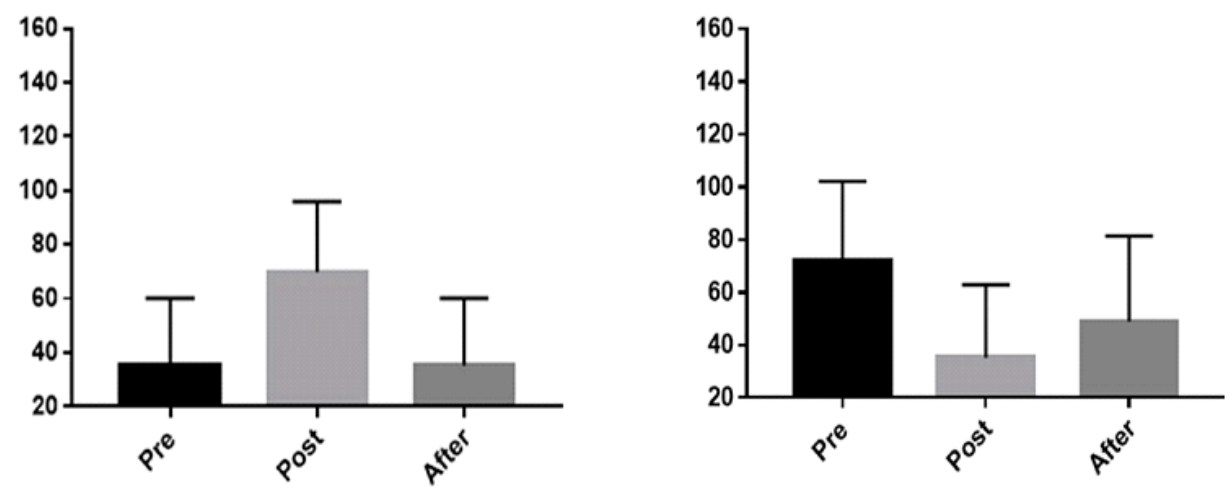

Figure A3. Results at the Negative and Positive Video. 


\section{References}

1. Eysenck, M.V.; Keane, M.T. Psicologia Cognitiva; Idelson-Gnocchi: Napoli, Italy, 2006; 109p.

2. Mérida-López, S.; Extremera, N.; Rey, L. Emotion-regulation ability, role stress and teachers' mental health. Occup. Med. (Lond.) 2017, doi:10.1093/occmed/kqx125.

3. Perciavalle, V.; Blandini, M.; Fecarotta, P.; Buscemi, A.; Di Corrado, D.; Bertolo, L.; Fichera, F.; Coco M. The role of deep breathing on stress. Neurol. Sci. 2017, doi:10.1007/s10072-016-2790-8.

4. Coco, M. The brain behaves as the muscle? Neurol. Sci. 2017, doi:10.1007/s10072-017-3014-6.

5. Coco, M.; Perciavalle, V.; Maci, T.; Nicoletti, F.; Di Corrado, D.; Perciavalle, V. The second-to-fourth digit ratio correlates with the rate of academic performance in medical school students. Mol. Med. Rep. 2011, 4, 471-476.

6. Coco, M.; Sarra Fiore, A.; Perciavalle, V.; Maci, T.; Petralia, M.C.; Perciavalle, V. Stress exposure and postural control in young women. Mol. Med. Rep. 2015, 11, 2135-2140, doi:10.3892/mmr.2014.2898.

7. Beedie, C.; Terry, P.; Lane, A. Distinctions between emotion and mood. Cogn. Emot. 2005, 19, 847-878, doi:10.1080/02699930541000057.

8. Panksepp, J. Affective Neuroscience: The Foundations of Human and Animal Emotion; Oxford University Press: New York, NY, USA, 1998.

9. Wierzbicka, A. Talking about Emotions: Semantics, Culture, and Cognition. Cogn. Emot. 1992, 6, $285-319$.

10. Verduyn, P.; Delvaux, E.; Van Coillie, H.; Tuerlinckx, F.; Van Mechelen, I. Predicting the duration of emotional experience: Two experience sampling studies. Emotion 2009, 9, 83-91.

11. Verduyn, P.; Van Mechelen, I.; Tuerlinckx, F. The relation between event pro-cessing and the duration of emotional experience. Emotion 2011, 11, 20-28.

12. Coates, J.M.; Gurnell, M.; Rustichini, A. Second-to-fourth digit ratio predicts success among high-frequency financial traders. Proc. Natl. Acad. Sci. USA 2009, 106, 623-628.

13. Caprara, G.V.; Barbaranelli, C.; Borgogni, L.; Vecchione, M. Big Five Questionnaire-2; Giunti O.S.: Firenze, Italy, 2008.

14. McNair, D.M.; Lorr, M.; Droppleman, L.F. Profile of Mood States; Giunti O.S.: Firenze, Italy, 1991.

15. Spielberger, C.D. STAI. State-Trait Anxiety Inventory - Forma Y; Giunti O.S.: Firenze, Italy, 1989.

16. Perciavalle, V.; Di Corrado, D.; Scuto, C.; Perciavalle, V.; Coco M. Anthropometrics related to the performance of a sample of male swimmers. Percept. Mot. Skills 2014, 118, 940-950, doi:10.2466/19.50.PMS.118k27w8.

17. Perciavalle, V.; Di Corrado, D.; Petralia, M.C.; Gurrisi, L.; Massimino, S.; Coco, M. The second-to-fourth digit ratio correlates with aggressive behavior in professional soccer players. Mol. Med. Rep. 2013, doi:10.3892/mmr.2013.1426.

18. Buscemi, A.; Rapisarda, A.; Platania, S.; Maida, F.; Brancati, D.; Petralia, M.C.; Di Nuovo, S.; Giustiniani, S.; Caldarella, L.; Blandino, M.G.; et al. The woman in pregnancy: Body care by knowing of alternative medicine. Acta Med. Mediterr. 2016, 32, 953.

19. Perciavalle, V.; Buscemi, A.; Borbone, C.; Catania, A.; Buscemi, B.; Petralia, M.C.; Puglisi, M.L.; Coco, L.S.G.; Coco, M. Exhaustive exercise and short term memory. Acta Med. Mediterr. 2016, 32, 23.

(C) 2017 by the authors. Licensee MDPI, Basel, Switzerland. This article is an open access article distributed under the terms and conditions of the Creative Commons Attribution (CC BY) license (http://creativecommons.org/licenses/by/4.0/). 